\title{
Effect of nanomaterial and media physicochemical properties on $\mathrm{Ag}$
}

\section{NM aggregation kinetics}

\author{
Kamelia Afshinnia ${ }^{1,2}$, Mithun Sikder ${ }^{1,2}$, Bo Cai ${ }^{3}$, and Mohammed \\ Baalousha $^{1,2, *}$
}

${ }^{1}$ Center for Environmental Nanoscience and Risk, Arnold School of Public Health, University South Carolina, Columbia, South Carolina 29208.

${ }^{2}$ Department of Environmental Health Sciences, Arnold School of Public Health, University South Carolina, Columbia, South Carolina 29208.

${ }^{3}$ Department of Epidemiology and Biostatistics, , Arnold School of Public Health, University South Carolina, Columbia, South Carolina 29208.

*Corresponding author: mbaalous@mailbox.sc.edu

Center for Environmental Nanoscience and Risk, Arnold School of Public Health, University South Carolina, Columbia, South Carolina 29208. Tel: 803-777-7177 


\section{Abstract}

Nanomaterial (NM) aggregation is a key process determining their environmental, fate behavior and effects. Nanomaterials are typically engineered to remain kinetically stable; however, in environmental and toxicological media, NMs are prone to aggregation. The aggregation kinetics of NM is typically quantified by measuring their attachment efficiency $(\alpha)$ and critical coagulation concentration (CCC). Several studies measured $\alpha$ and CCC for Ag NMs with a major focus on investigating the effects of ionic strength, ion valency and natural organic matter, with few studies investigating other environmental factors such as light and dissolved oxygen and none investigating the effect of particle size, buffer type and concentration, or surface coverage by capping agent. A survey of recent research articles reporting CCC values for Ag NMs reveals substantial variation in experimental conditions and particle stability with CCC values of monovalent and divalent counterions covering a wide range (ca. 25 to infinity for monovalent counterions and 1.6 to infinity for divalent counterions).

Here, we rationalize the differences in the CCC values for Ag NMs based on the variability in the experimental conditions, which includes NM and medium physicochemical properties. Capping agents determines NM stability mechanism with citrate, sodium dodecyl sulfate (SDS), and alginate stabilizing NM by electrostatic mechanism; whereas polyvinylpyrrolidone (PVP), casein, dextrin, tween, branched polyethyleneimine (BPEI), and Gum Arabic stabilizing NMs by steric mechanisms. The CCC values for Ag NMs with different capping agents follow the order citrate $~$ alginate $\sim$ SDS $<$ casein $<$ dextrin $<$ PVP $<$ tween < branched polyethyleneimine (BPEI) gum Arabic. For charge stabilized Ag NMs, the CCC increases with the decrease in NM size and buffer concentration and decreases with light irradiation. For sterically stabilized PVP-Ag NMs, the CCC increases with the coating concentration/surface coverage and completely coated Ag NMs do not undergo aggregation even at high salt concentrations. 


\section{Introduction}

Understanding NM stability, fate and behavior in environmental and toxicological media is crucial to allow for comprehensive environmental risk assessment of $\mathrm{NMs}^{1}$. Aggregation is one of the key processes, among others, determining their environmental fate, behavior, uptake, and effects ${ }^{1-3}$. Furthermore, understanding the interplay of NM- and medium- physicochemical properties and their aggregation behavior under conditions of ecotoxicological and environmental media is required for an adequate interpretation of ecotoxicological test outcomes, fate and behavior studies, to generate parameters (e.g. attachment efficiency and critical coagulation concentration, $\alpha$ and CCC, respectively) ${ }^{3}$ for environmental fate models ${ }^{4-6}$, and for comparison among different studies.

One of the quantitative methods to assess the stability of any dispersion is to measure the attachment efficiency $(\alpha)$ - the inverse of the stability ration $(W)$ - and the CCC, which is the minimum electrolyte concentration required to fully destabilize the dispersion ${ }^{7}$. The $\alpha$ and $C C C$ parameters of a specific counterion is commonly described by the classical DLVO theory on the basis of a static force balance. The DLVO theory attributes the interaction between two identical particles to van der Waals attraction and the electrical double layer repulsion ${ }^{8,9}$. A substantial progress in the understanding of the stability phenomena has been made since the introduction of the DLVO theory. Additionally, numerous studies have shown the importance of other interaction forces such as steric forces, hydration, etc ${ }^{10}$. The CCC can, however, also be estimated from a kinetic point of view by studying the process of colloidal coagulation, or from a dynamic point of view by considering colloidal transport in nonequilibrium systems where other processes such as diffusion and the influence of gravity come into play $^{11}$. Several Ag NM aggregation kinetics' experimental studies have focused on investigating the effect of ionic strength, ion valency, $\mathrm{pH}$ and natural organic matter (NOM) concentration on the stability of Ag NMs $(C C C \text { and } \alpha)^{12-16}$. However, wide discrepancies can be found in the literature among the reported CCC and $\alpha$ values (Table S1). The CCC values of monovalent and divalent counterions for $\mathrm{Ag}$ 
NMs found in the literature cover a wide range ( $c a .25$ to $\alpha$ for monovalent counterions and 1.6 to $\alpha$ for divalent counterions). These differences are likely to be attributed to the differences in the experimental conditions among the different studies including: 1) method used to study NM aggregation kinetics (dynamic light scattering, DLS, UV-vis, add others) ${ }^{16}, 2$ ) physicochemical properties of the NMs (e.g. size, shape, surface coating, etc. $)^{17,18}$, and 3) media chemistry (buffer type and concentration) ${ }^{19}$.

There is currently a limited, and often contradictory, knowledge on the effect of intrinsic properties of Ag NMs (and other NMs in general) and medium, other than ionic strength, ion valency and NOM on the stability of Ag NMs. For instance, recent studies reported contradictory results on the dependence of $\mathrm{CCC}$ on NM size including a decrease with the decrease in NM size (e.g. hematite ${ }^{20}, \mathrm{TiO}_{2}$ $\left.{ }^{18}\right)$, an increase with the decrease in NM size $\left(\right.$ e.g. $\mathrm{CdSe}^{21}$ ) or independence of CCC of NM size (e.g. Au $\mathrm{NMs}^{22}$ ). However, no studies in the literature investigated the effect of Ag NMs size on their stability. In addition, whereas some studies reported a linear correlation between the CCC and NM primary particle size (e.g. anatase $\left.\mathrm{TiO}_{2}{ }^{18}\right)$, other studies found that the $\mathrm{CCC}$ correlated better with NM specific surface area (e.g. anatase $\mathrm{TiO}_{2}{ }^{18}, \mathrm{CdSe}^{21}$ ). Other studies reported an important role of stabilizing agent ${ }^{21}$, impurities introduced during synthesis process $^{23}$, etc. on NM stability. These studies suggest that material properties such as particle size, capping ligand, impurities, and other properties are important parameters affecting NM stability. However, a systematic review/analysis of the role of intrinsic properties of Ag NM on their aggregation kinetics is lacking.

In this context, this article aims to 1 ) investigate the relationship between the $C C C$ values for $\mathrm{Ag}$ NMs and the parameters affecting Ag NM aggregation behavior and kinetics, and 2) rationalize the discrepancies in the reported CCC values for Ag NMs in the literature. This article also provides a critical discussion on deviations from DLVO theory due to NM specific properties. 


\section{Methods}

Data collection

Data on Ag NM properties (size, zeta potential, coating type and concentration, and NM concentration), water physicochemical properties $(\mathrm{pH}$, buffer concentration, ion type and concentration, concentration and type of NOM) and CCC were extracted from research articles reporting Ag NM aggregation kinetics' (11 articles) experiments and deriving CCC values (55 measurements) and tabulated (Table S1 and 2). Ag NMs were selected for this review as they are the most widely studied NMs in the literature with the largest number of articles reporting CCC values under variable NM and media properties.

\section{Data analysis}

The collected data for Ag NMs were separated into electrostatically and sterically stabilized NMs to facilitate data discussion, interpretation and rationalization of differences. The relationships between NM physicochemical properties, media physicochemical properties and CCC were examined for both electrostatically and sterically stabilized NMs by regression analysis. Due to the relatively small sample size (range 4-18) and the non-linear association (e.g. Fig 1 and Fig 4), the Spearman's non-parametric rank correlation test was used to test if there exist significant relationships between NM physicochemical properties/media physicochemical properties and CCC under various experimental conditions. The statistical significance was set as $p$-value $<0.05$.

\section{Zeta potential measurement}

Citrate-coated Ag NMs were synthesized and characterized using a previously reported method ${ }^{24}$. The properties of these citrate-coated Ag NMs are reported elsewhere ${ }^{25}$. Briefly, the size and electrophoretic mobility (EPM) of AgNMs were measured by dynamic light scattering (DLS) and laser Doppler electrophoresis, respectively using a Malvern Zetasizer NanozS Instrument (Malvern, USA). According to DLS, the z-average hydrodynamic diameter $\left(z-d_{h}\right)$ and polydispersity index of synthesized citrate-coated $\mathrm{Ag} \mathrm{NMs}$ in stock suspension were $24.48 \pm 0.10 \mathrm{~nm}$ and $0.21 \pm 0.05$, 
respectively. The zeta potential of the synthesized $\mathrm{Ag} \mathrm{NMs}$ was $-41.7 \pm 2.0 \mathrm{mV}$ at approximately $\mathrm{pH} 7.0$ using Smoluchowsky approximation. The standard deviation reported here shows the stability of the replicate measurements and was determined from 5 replicates of size and 10 replicates of zeta potential.

Polyvinylpyrrolidone coated Ag NMs (PVP-Ag NMs) with an increased number of PVP molecules per Ag NM unit surface area were prepared to evaluate the effect of PVP surface coverage on the zeta potential of PVP-Ag NMs. Briefly, PVP-Ag NMs were prepared by ligand exchange approach using citratecoated Ag NMs as precursor. Aliquots of Citrate-coated Ag NMs (Cit-Ag NMs) were converted into PVPAg NMs by adding 4-16 molecules of PVP per $\mathrm{nm}^{2}$ surface area of cit-Ag NMs under vigorous stirring (e.g. $700 \mathrm{rpm}$ ) for at least 1 hour and then cooled overnight at $4^{\circ} \mathrm{C}$. The electrophoretic mobility of $\mathrm{Ag} \mathrm{NMs}$ was measured by laser Doppler electrophoresis using a Malvern Zetasizer NanoZS Instrument (Malvern, USA). The $\mathrm{pH}$ of all Ag NM suspension was routinely measured and was approximately 7.0 \pm 0.2 .

\section{DLVO calculations}

Classical DLVO calculations were performed to underpin the observed results according to the equations presented $\mathrm{in}^{15,26}$ and according to the following assumptions: (i) ionic strength of $10^{-3} \mathrm{M}$ monovalent electrolyte, (ii) the zeta potential is constant at $-42 \mathrm{mV}$, (iii) steric stabilization induced by capping agents is not considered.

\section{Results and discussion}

A survey of recent research articles in the field reveals substantial variation in experimental conditions (Table S1). Variations include NM size, concentration, surface coating type and concentration, buffer concentration, electrolyte type and concentration, dissolved oxygen (DO), and light. For instance, Z-average hydrodynamic diameter ranged from 21 to $107.5 \mathrm{~nm}$, number average NM size ranged from 5.4 to $82.0 \mathrm{~nm}$, NM concentration ranged from 0.4 to $24.5 \mathrm{mg} \mathrm{L}^{-1}$, buffer concentration ranged from 0 to $0.15 \mathrm{mM} \mathrm{NaHCO}_{3}$, and capping agents includes citrate, alginate, SDS, casein, dextrin, 
PVP, tween, BPEI, and gum Arabic. Small variations in reaction conditions may be largely irrelevant or may fundamentally change the fate and behavior of NMs such that comparisons between datasets become challenging. The CCC values of monovalent and divalent counterions for Ag NMs found in the literature cover a wide range (ca. 25 to infinity for monovalent counterions and 1.6 to infinity for divalent counterions; Table S1). Below we rationalize the differences in the CCC values based on the variability in the experimental conditions, which includes NM and medium physicochemical properties.

\subsection{Capping agent}

Overall, the CCC values of Ag NMs with different capping agents follow the trend citrate alginate $\sim$ SDS $<$ casein $<$ dextrin $<$ PVP $<$ tween $<$ BPEI $\sim$ gum Arabic (Table S1). The capping agent is the factor that determines NM stabilization mechanism ${ }^{2,27}$. Physicochemical properties of the capping agent have a greater influence on the aggregation behavior of functionalized Ag NMs than either core composition or their size (Table $\mathbf{S 1})^{22}$. Some capping agents such as citrate, SDS, and alginate stabilize

NM by electrostatic mechanism ${ }^{16,28-32}$; whereas other capping agents such as PVP, casein, dextrin, tween, branched polyethyleneimine (BPEI), and Gum Arabic stabilize NMs by steric mechanisms ${ }^{29,32,33}$. Generally, the CCC of charge stabilized Ag NMs are lower than those of sterically-stabilized Ag NMs, and some sterically stabilized Ag NMs are kinetically stabile at even very high salt concentrations (ca. up to $10 \mathrm{M} \mathrm{NaNO}_{3}$ and $8 \mathrm{M} \mathrm{CaCl}_{2}$ ). The dependence of the $\mathrm{CCC}$ values on $\mathrm{Ag} \mathrm{NM}$ and media properties are discussed for the two categories based on the stability mechanisms of Ag NMs: charge- and stericallystabilized NMs.

\subsection{Behavior of Charge-Stabilized NMs}

For charge-stabilized Ag NMs (e.g. bared, citrate, SDS, and alginate coated), the CCC values show a wide discrepancy ranging from 30 to $122 \mathrm{mM} \mathrm{NaCl}, 25$ to $200 \mathrm{mM} \mathrm{NaNO}_{3}, 1.6$ to $2.2 \mathrm{mM} \mathrm{CaCl}_{2}$ and 1.7 to $5 \mathrm{mM} \mathrm{Ca}\left(\mathrm{NO}_{3}\right)_{2}$. The differences in the $\mathrm{CCC}$ values are likely due to differences in $\mathrm{NM}$ properties (e.g. 
NM size, concentration, capping agent concentration) and medium chemistry (e.g. counter ion types, buffer concentration, etc.). Charge-stabilized NMs owe their stability to particle charge, which results in the formation of diffuse double layer around NMs. Any constituent which influences the surface charge will also change NM stability. The most profound effect is exercised by ions of charge opposite to that of NMs, which result in charge screening and shrinkage of the diffuse double layer thickness. Yet counterion charge is not the only factor influencing the stability of a given NM. Size, shape, degree of hydration, character of the ligands in complexes and other parameters play important roles ${ }^{34}$. However, little attention has been given to the effects of these factors on NM aggregation kinetics in the literature.

\section{Counterion and natural organic matter}

The effects of counterions, $\mathrm{pH}$ and NOM on $\mathrm{Ag} \mathrm{NM}$ aggregation kinetics are the most widely studied and understood (see Table S1), and therefore are only briefly summarized here. Divalent counterions (cations or anions) are more efficient in screening NM surface charge than monovalent counterions, in good agreement with Schulze-Hardy rule ${ }^{35-37}$. For a typical electrostatically stabilized NM, the CCC of an electrolyte depends strictly on the counterion charge, as long as the adsorption of the coion or the counterions on the surface is negligible. This is the condition when the Schulze-Hardy rule is obeyed. If an ion adsorbs sufficiently strongly at concentrations lower than the CCC, the Schulze-Hardy rule does not apply ${ }^{34}$. The majority of studies on electrostatically stabilized Ag NMs reported CCC values for monovalent and divalent electrolytes $\left(c a . C C C_{m o n o} / C C C_{d i}=8-43^{30,38-41}\right.$ ) within the boundaries ( $c a$. $\left.\mathrm{CCC}_{\text {mono }} / \mathrm{CCC}_{\mathrm{di}}=4-64\right)$ of the Schulze-Hardy rule ${ }^{35,37}$.

Natural organic matter (typically Suwannee River fulvic and humic acids) increases the CCC of Ag NMs by 1.4-4.3 folds (Table S1 and 2). This is because NOM replaces the capping agent (typically citrate) and impact electrosteric stabilization of $\mathrm{Ag} \mathrm{NMs}^{42}$. Additionally, several studies have demonstrated that NOM results in a reduction in the Hamaker constant ${ }^{16,30,43}$. 


\section{Size}

The effect of size on CCC and $\alpha$ of Ag NMs has not been investigated explicitly in any study in the literature. Taking all CCC values reported in the literature for charge stabilized Ag NMs, clear relationship between NM size and CCC can be determined. For monovalent electrolytes, the CCC shows a strong association between NM hydrodynamic diameter measured by DLS ( $P$ value $=0.0075$ and 0.00499 for $\mathrm{NaCl}$ and $\mathrm{NaNO}_{3}$ counterions; Figure 1a), or primary NM size measured by microscopy techniques ( $\mathrm{P}$ value $=0.0735$ for $\mathrm{NaNO}_{3}$ counterions; Figure 1c). The $\mathrm{CCC}$ increase with the decrease in NM size, with a sharp increase in the CCC at approximately a z-average hydrodynamic diameter of $50 \mathrm{~nm}$ and a core size of approximately $20 \mathrm{~nm}$. This behavior is in agreement with a previous study on citrate-Au NMs ${ }^{44}$, where Frens observed a marked increase in the CCC (from 5 to $70 \mathrm{mM} \mathrm{NaNO}_{3}$ ) of citrate-Au NMs with the decrease in particle size (from 70 to $7.5 \mathrm{~nm})^{44}$. They used this phenomenon to fractionate a mixture of particles ( 7.5 and $80 \mathrm{~nm}$ ) according to particle size by coagulating the larger particles by addition of 30 $\mathrm{mM} \mathrm{NaNO}_{3}$. Despite the strongly negative association between the $\mathrm{CCC}$ and particle size for monovalent electrolytes (Figure 1 a and c, Table S3), no clear trend was observed between CCC and Ag NM size for divalent electrolytes (Figure $\mathbf{1} \mathbf{b}$ and $\mathbf{d}$, Table S3). The Spearman's correlation tests were presented in Table S3.

The increase in the CCC with the decrease in Ag NM size seems to be in contradiction with the general notion that the interaction energies of NM during slow coagulation should be proportional with the particle size and that, therefore, larger particles are expected to be more stable under equal experimental conditions ${ }^{45}$. However, this notion applies only for slow coagulation regime (reaction limited aggregation regime; Figure 2) and does not affect the CCC value. Figure 2 illustrates that according to DLVO theory, under RLA regime, the attachment efficiency decreases with the increase in NM size, but the CCC remains constant. This argument has been misleadingly used in several publications to justify the increase in CCC with the increase in NM size ${ }^{18}$. 
Recent studies reported contradictory results on the dependence of CCC on NM size including a decrease with the decrease in NM size (e.g. hematite $\left.{ }^{20}, \mathrm{TiO}_{2}{ }^{18}\right)$, an increase with the decrease in NM size (e.g. $\mathrm{CdS}^{21}$ ) or independence of CCC of NM size (e.g. Au NMs ${ }^{22}$ ). Similarly, earlier studies on colloid stability reported contradictory results including a decrease ${ }^{46-48}$, an increase ${ }^{20}$, an increase followed by a decrease ${ }^{49,50}$, as well as an independence of colloid stability ${ }^{51}$ on particle size. These contradictory results were attributed, at least partly, to deviations from sphericity and contributions of surface irregularities rather than to differences in particle size ${ }^{17}$. Other explanations such as distribution of surface potential, hydrodynamic interaction, dynamics of interaction, deposition in secondary minimum, and surface roughness have been discussed in details elsewhere ${ }^{51}$.

For instance, some studies ${ }^{17,49,52}$ accounted for the decrease in CCC with the increase in particle size by considering secondary minimum flocculation in the available models ${ }^{53}$, where small particles aggregate in primary minimum, whereas large particles aggregate in secondary minimum ${ }^{54}$. Secondary minimum in the total energy of interaction profile can occur at moderate to large separations (usually larger than several nanometers). For a given medium properties, the depth of the secondary minimum increases with particle size (Figure 3). Coagulation of Brownian particles in secondary minimum is possible, in principle, if the resultant of the force from the thermal energy of the particles and the fluid drag is insufficient to drive the particles out of the secondary minimum. Indirect evidence for this phenomenon has been reported by numerous investigators ${ }^{49,55,56}$. For instance, the CCC of hematite particles (ca. 74 to $704 \mathrm{~nm}$ ) as a function of particle size shows a minimum at a diameter of about 400 $\mathrm{nm}$. The small particles probably coagulate in the primary minimum, whereas for the large particles secondary minimum flocculation is dominant ${ }^{17}$. The increase in CCC for $d>400 \mathrm{~nm}$ was explained by shear induced aggregate disruption for electrolyte concentrations below the CCC.

Another reason for such a deviation from classical-DLVO prediction of size-independent CCC values is the assumptions used in DLVO theory. The derivation of the DLVO theory was based on the 
assumptions that the thickness of the electrical double layer $(\kappa)$ surrounding a charged particle in an electrolyte solution is much smaller $(r)$ than the linear size of the particle $(k r » 1)$, and the electrical interaction energy can be estimated through the superposition principle. The former implies that the CCC is independent of the size of a particle, and the latter assumes that two interacting particles can be considered independently. Hsu and Liu, examined the assumptions made by the DLVO theory by investigating the electrical interaction between two spherical particles as a function of particle size at constant surface potential and considering the case that the thickness of the electrical double layer is not necessarily much smaller than the radius of a particle ${ }^{57}$. They concluded that, at constant surface potential, if the radius of a particle is $>1 \mu \mathrm{m}, \mathrm{CCC}$ is independent of particle size; whereas for smaller particles, the CCC increases with the decrease in particle size ${ }^{57}$. This deviation was attributed to the application of the superposition principle in the evaluation of the electrical interaction energy ${ }^{57}$.

Furthermore, the contradictory results in the literature regarding the effect of NM size on the CCC values can be rationalized by taking into account the variability in NM surface charge (e.g. zeta potential). It is clear that in the studies reporting a decrease in CCC with the increase in NM size, the NMs are characterized by a narrow range of surface potential (ca. zeta potential of $-33 \pm 3$ to $-35 \pm 5$ for $\mathrm{CdSe}_{\mathrm{NMs}}^{21}$ ); whereas the studies reported an increase in CCC with the increase in NM size, or a sizeindependence of CCC, NMs are characterized by variable zeta potential ${ }^{18,20}$.

For instance, the similarity in the CCC values of 30 and $100 \mathrm{~nm}$ Au NMs reported by Liu et al can be attributed to the differences in the zeta potential of the pristine particles (ca. -42 and -50 for the 30 and $100 \mathrm{~nm}$ NMs, respectively), which might compensate for the differences in NM size ${ }^{22}$. That is, the increased zeta potential of the $100 \mathrm{~nm}$ could compensate for the expected reduction in CCC value, thus $\mathrm{CCC}$ value for the 30 and 100 is the same. Similarly, the surface charge of $\mathrm{TiO}_{2} \mathrm{NMs}$ decrease (become more negative) with the increase in NM size, thus the increase in the CCC value with the increase in NM $\operatorname{size}^{18}$. The decrease in hematite NM CCC $(70 \pm 6,54 \pm 9$, and $45 \pm 4)$ with the decrease in NM size $(65,32$ 
and $12 \mathrm{~nm}$ ) can be attributed to the decrease of zeta potential of NMs (ca. 33 $\pm 2.7,24 \pm 2.9$, and 15.1 \pm 1.7 , respectively) as particle size decreases ${ }^{20}$. Therefore, in these studies ${ }^{18,20,22}$, the correlation between NM size and CCC could be influenced by the variability of the surface charge, and cannot be attributed solely to NM size.

It is noteworthy the zeta potential of all charge-stabilized Ag NMs (Table S1) varies within a tight range (ca. -35 to -48$)$, except two NMs (-22 to -26.5$)$, which can explain the differences in the trend of CCC with size for charge stabilized Ag NMs compared to other studies ${ }^{18,20}$. Additionally, larger number of NMs is considered in this review allowing accounting for the variability in the experimental conditions.

\section{Buffer concentration}

Buffers are commonly used to maintain a constant $\mathrm{pH}$ during a reaction, but their presence can result in substantial changes in surface chemistry ${ }^{58}$ and NM stability ${ }^{19,59}$. Few studies investigated the impact of buffer concentration on NM aggregation. For instance, increasing 3Morpholinopropanesulfonic acid (MOPS) buffer concentration resulted in an increased goethite NMs aggregate size, which was attributed to sorption of MOPS molecules on the surface of goethite NMs accompanied by a reduction in surface charge ${ }^{19}$. Furthermore, the buffer identity greatly affects the aggregation state of goethite NMs. Goethite NMs suspended in solution containing $1 \mathrm{mM} \mathrm{Fe}{ }^{2+}$ and HEPES (4-(2-hydroxyethyl)-1-piperazineethanesulfonic acid) buffer were the most dispersed, and the aggregate sizes were 3-5 times larger for goethite NMs suspended in MOPS and Triethanolamine (TEA) buffers $^{19}$. The stability of hematite particles decrease in the presence of phosphate anions ${ }^{59}$. Therefore, while buffers enable adequate maintenance of $\mathrm{pH}$, their presence is not passive with regard to NM surface and aggregation kinetics. However, there is no single study that investigated the impact of buffer type and concentration on the aggregation kinetics of NMs in the literature.

Buffered (carbonate buffer) Ag NMs have lower CCC values compared to unbuffered Ag NMs and the CCC values decrease with the increase in buffer concentration (Table S1 and Figure 4a). The 
Spearman's correlation test (Table S3) showed a significant negative correlation between buffered Ag $\mathrm{NMs}$ and the $\mathrm{CCC}$ values (Pvalue $=0.0070$ for $\mathrm{NaCl}$ ). For $\mathrm{NaNO}_{3}$, the Spearman's correlation test (Table S3) did not show a significant negative correlation between buffered Ag NMs and the CCC values (Pvalue $=0.8089)$, which is likely due to the small number of samples and the effect of NM size on the CCC in addition to the effect of buffer. These observations suggest that $\mathrm{pH}$ buffers are likely to play an important role in controlling NM stability and need to be further investigated. This is consistent with the high affinity of carbonate anions to silver $\left(K s p\right.$ at $\left.25^{\circ} \mathrm{C}=8.46 \times 10^{-12} \mathrm{M}^{2}\right)$, which may result in the formation of $\mathrm{Ag}_{2} \mathrm{CO}_{3}$ layer on the surface of $\mathrm{Ag} \mathrm{NMs}$, altering $\mathrm{NM}$ surface charge and Hamaker constant and thus Ag NM stability. At pH 7, the zeta potential of Ag NMs increases (become less negative) with the decrease in NM concentration (1 to $100 \mu \mathrm{M})$ in $10 \mathrm{mM}$ carbonate concentration ${ }^{60}$. Concurrently, $\mathrm{Ag}$ NM aggregate size increases with the decrease in NM concentration. This behavior can be attributed to the increased sorption of $\mathrm{CO}_{3}{ }^{-}$anions on the surface of $\mathrm{Ag} \mathrm{NMs}$ at lower NM concentrations due to the increased abundance of $\mathrm{CO}_{3}{ }^{-}$anions compared to $\mathrm{NM}$ total surface area.

\section{Chemical interactions of NMs with water constituents}

Some medium constituents have very high chemical affinity to a wide spectrum of NMs. Taking silver as an example; $\mathrm{Ag}_{2} \mathrm{~S}$ have a stability constant of $8 \times 10^{-51} \mathrm{M}^{2}, \mathrm{Ag}_{3} \mathrm{PO}_{4}$ of $8.9 \times 10^{-17} \mathrm{M}^{2}, \mathrm{Ag}_{2} \mathrm{CO}_{3}$ of $8.46 \times 10^{-12} \mathrm{M}^{2}$, etc. These strong interactions of NMs with these constituents are likely to form a surface layer of Ag complexes, resulting in the formation core-shell NMs, and thus controls NM surface charge and aggregation in these media and some emerging studies have demonstrated these effects ${ }^{61}$.

The chemical sorption of macromolecules, anions, buffers, etc. has been widely ignored in studying NM aggregation kinetics. However, some studies have reported the effect of some of these parameters on NM/aggregate size ${ }^{61-68}$. For instance, addition of cystine to citrate-stabilized Ag NMs results in NM concentration-dependent shift in the CCC, where the CCC as $\mathrm{mM}$ cystine decrease with the decrease in Ag NM concentration ${ }^{31}$. Organic compounds containing both thiol and amine groups strongly promote 
the aggregation of citrate-coated Au NMs due to their cooperative functionalities ${ }^{66}$. Three features of organic compounds could influence citrate-stabilized Au NMs (and similarly Ag NMs); the presence of a sulfur atom, which can form covalent bonds with gold atoms, the presence of ionizable functional groups, and the sign (+ or -) of ionizable functional groups ${ }^{66}$.

Furthermore complexation of the counterion in electrolyte mixtures may result in an increase in the measured CCC values. For instance, Matijevic and Stryker (1966) reported an increase in CCC of Aluminum ion for negatively charged silver iodide sols if the sol is acidified by sulfiric acid; whereas this behavior was not observed when nitric acid or perchloric acids are used to lower the $\mathrm{pH}^{69}$. This behavior was attributed to the formation of $\mathrm{AlSO}_{4}{ }^{+}$complexes under acidic conditions resulting in the reduction in the concentration of $\mathrm{Al}^{3+}$ ions ${ }^{70}$; according to Schulze-Hardy rule monovalent ions are orders of magnitude less efficient in causing particle aggregation compared with trivalent ions. These two studies reveal the importance of considering counterion speciation in interpreting the variation in CCC.

\section{Dissolved oxygen}

Dissolved oxygen (DO) in the aqueous environments tend to oxidize metallic $\mathrm{NMs}$ such as $\mathrm{Ag}$ NMs and QDs ${ }^{71-74}$. The oxidation invokes several complex physicochemical processes that may occur simultaneously such as formation of a surface oxide layer $\left(\right.$ e.g. $\left.\mathrm{AgO}^{75}\right)$, release of the metallic cations $^{72,75,76}$, complexation and speciation of metallic cations with aqueous components such as NOM), and aggregation ${ }^{71,72,77}$. For instance, the aggregation rate of Ag NMs is much faster (e.g., 3-8 times depending on the primary particle size) in the presence of DO than those without DO. The hydrodynamic diameter of Ag NMs had a linear growth within the initial 4-6h and after the linear growth, the hydrodynamic sizes became random for Ag NMs in the presence of DO, whereas in the absence of DO the hydrodynamic sizes grew smoothly and steadily ${ }^{71}$. The hydrodynamic diameter of Ag NMs increased more drastically and randomly in the presence of $\mathrm{DO}$, probably due to $\mathrm{Ag}^{+}$ion release and particle debris formation; whereas in the absence of DO, the hydrodynamic diameter increased relatively slower and 
linearly. The increased aggregation of $\mathrm{Ag} \mathrm{NMs}$ in the presence of DO was attributed to an unknown mechanism such as surface energy changes due to adsorption of $\mathrm{Ag}^{+}$or surface oxidation ${ }^{71}$. Surface oxidation of $\mathrm{Ag}$ NMs has been reported to increase the zeta potential of Ag NMs (become less negative $)^{78}$, which could be due to the local increase in $\mathrm{Ag}^{+}$at the surface of $\mathrm{Ag} \mathrm{NMs} \mathrm{during}$ the dissolution process, resulting in surface charge neutralization and Ag NM aggregation. Furthermore, NM aggregation can be related to the degradation/detachment of surface coating in the presence of oxygen. For instance, the destabilization of $\mathrm{Ag} \mathrm{NMs}, \mathrm{CdS} \mathrm{NMs}$ and QDs was found to be related to the detachment/degradation of surface coating in the presence of oxygen, compression of EDL and surface energy changes ${ }^{72,77,79}$. In contrast, anoxic and anaerobic conditions exert low redox potentials, which inhibit oxidation and consequently lead to different aggregation kinetics ${ }^{72,77}$.

\section{Light}

Light exposure is another important environmental factor affecting NM aggregation behavior and other NM physicochemical processes ${ }^{72,80-82}$. Light irradiation such as UV, Xenon lamp, solar and even fluorescent light has been shown to promote NM surface oxidation, dissolution and aggregation ${ }^{80,83,84}$. For instance, the CCC of $\mathrm{NaNO}_{3}$ for citrate-Ag NMs decreased with 3-day exposure to UV radiation compared with unexposed Ag NMs. The CCC values for fresh, 3 day UVA (320-400 nm)- and 3 day UVB (280-320 nm)-aged Ag NMs were 161.4, 37.8 and $21.4 \mathrm{mM} \mathrm{NaNO}_{3}$. This decrease in the CCC was concurrent with the increase in the $\zeta$ potential (become less negative) after exposure to UVA and UVB ${ }^{83}$.

Similarly, sunlight irradiation resulted in aggregation of polymer coated Ag NMs (e.g. 6 and 25 $\mathrm{nm}$ coated with gum Arabic and $25 \mathrm{~nm}$ coated with PVP) to different degrees, depending on surface coating and the UV content of the light. The stability of PVP-Ag NMs was generally better than that of Gum Arabic-Ag NMs ${ }^{82}$. The UV content of the sunlight is identified to be the driving force of NM aggregation, and the strong oscillating dipole-dipole interaction is believed to be the origin of destabilization $^{82}$. In parallel, Ag NM toxicity was greatly reduced after sunlight irradiation ${ }^{82}$. The 
aggregation rate of bared-, citrate-, and PVP-Ag NMs followed the order UV-365 > xenon lamp > UV254> dark for the same type of Ag NMs, which indicated that the wavelength and photo-energy of the light irradiation influence Ag NM aggregation kinetics ${ }^{77}$. The aggregation rate was highest for bare-Ag NMs followed by citrate Ag NMs and PVP Ag NMs.

\subsection{Behavior of Sterically Stabilized NMs}

For sterically-stabilized Ag NMs, there is a wide discrepancy in the reported CCC values ranging from $111.5 \mathrm{mM} \mathrm{NaCl}$ to no CCC up to $10 \mathrm{M} \mathrm{NaCl}, 530$ to no CCC up to $10 \mathrm{M} \mathrm{NaNO}_{3}$, and 4.9 to No CCC up to $8 \mathrm{M} \mathrm{CaCl}_{2}$ (Table S1). This can be attributed to the surface coverage of Ag NMs by the capping agent molecules, where high capping agent concentrations may result in full coverage and thus full steric stabilization, whereas lower capping agent concentrations may result in partial coverage and thus electrosteric stabilization (e.g. partial steric stabilization of Ag NMs). Evidence supporting this hypothesis can be drawn from studies on other surface coating such as human serum albumin (HSA) ${ }^{67}$ and gum $\operatorname{gar}^{85}$. For instance, increased concentration of human serum albumin (HSA) protein resulted in a reduced attachment efficiency of citrate-coated $\mathrm{Ag} \mathrm{NM}$ in $\mathrm{K}_{2} \mathrm{SO}_{4}$ electrolyte. The attachment efficiency decreased with increasing HSA concentration until the attachment efficiency reached zero which coincided with the HSA concentration required to form a monolayer on the surface of $\mathrm{Ag} \mathrm{NMs}{ }^{67}$. They described the attachment efficiency as a function of surface coverage $\left(\alpha=(1-\theta)^{2}\right.$, where $\theta$ is the surface coverage) assuming agglomeration between two colliding particles can only occur when two unoccupied surface sites collide ${ }^{67}$. Similarly, increased concentration of guar gum (a natural, neutrally charged, nonionic, water-soluble polysaccharide; 0 to $4.0 \mathrm{~g} \mathrm{~L}^{-1}$ ) enhanced the stability of zero valent iron NMs (1.5 $\left.\mathrm{g} \mathrm{L}^{-1}\right)$; that is resulted in decreased aggregate size (ca. from 500 to $\left.200 \mathrm{~nm}\right)^{85}$.

Interestingly, the CCC of monovalent electrolytes $\left(\mathrm{NaCl}\right.$ and $\left.\mathrm{NaNO}_{3}\right)$ for PVP-Ag NMs decreases with the increase in zeta potential (Figure 5), which can be used to explain the variability in the reported CCC for PVP-Ag NMs. PVP molecules have little to no charge and fully coated Ag NMs have been 
reported in the literature to have a zeta potential of approximately $-10 \mathrm{mV}^{86}$ similar to the value reported by El Badawy $2012^{29}$, which coincides with a full steric stabilization of the NMs and absence of a CCC value. On the other hand, the studies by Zhang $2012^{28}$, Huynh $2011^{30}$ and Lin et al ${ }^{33}$ reported significantly high zeta potential values, which are likely to be due to partial surface coating of Ag NMs by PVP molecules and thus these particles might be partially charge and partially sterically stabilized, with those studied by Huynh $2011^{30}$ being more charge stabilized, thus the lower CCC value. This is in good agreement with the concentrations of PVP used to stabilize NMs in these three studies; that is PVP concentrations follow the order Huynh $2011^{30}<$ Zhang $2012^{28}<$ El Badawy $2012^{29}$. Coating zero valent iron NMs with guar gum (a natural, neutrally charged, nonionic, water-soluble polysaccharide; $0.5 \mathrm{~g} \mathrm{~L}^{-1}$ ) resulted in a nearly neutrally charged NMs at all pHs (2-12) compared to the bared NMs which were positively charged at low $\mathrm{pHs}$ and negatively charged at high $\mathrm{pHs}$ with an isoelectric point at approximately $\mathrm{pH} 7.0^{85}$. These observations highlight the importance of characterizing the surface coverage of NMs by the capping agent.

To further support the hypothesis on the role of surface coverage on zeta potential and ultimately on CCC observations, the zeta potential for in house synthesized cit-Ag NMs coated with variable number of PVP molecules (e.g. 4-16) by ligand exchange is presented in Figure $\mathbf{5 b}$, and demonstrates the decrease in zeta potential magnitude with the increase in the number of PVP molecules on the surface of Ag NMs.

No CCC value was measured for Gum Arabic coated Ag NMs (GA-AgNMs) due to steric stabilization $^{32}$. GA is a polymer with a high molecular weight $\left(5 \times 10^{4}\right.$ to $\left.8 \times 10^{6} \mathrm{~g} \mathrm{~mol}^{-1}\right)$ with a strong stabilization power ${ }^{33,87}$. However, this steric stabilization effect disappeared at long experimental times and enhanced $\mathrm{NaCl}$ concentrations ${ }^{32}$.

Clearly, fully sterically stabilized Ag NMs do not undergo aggregation. Thus, the polymer effect is the key factor determining their stability and NM and medium physicochemical properties do not play 
any role. However, the key question that remains unanswered is the importance of the capping agent following release of NMs to natural waters and their interaction with other medium constituents that may replace the capping agent due to their high affinity to the NM under consideration. For instance, cystine has been shown to impart aggregation of citrate, PVP and PEG-coated Ag NMs, which was attributed to the higher affinity of cystine than the capping agent to $\mathrm{Ag} \mathrm{NMs}{ }^{31}$.

\section{Conclusions}

This review highlights the role of $\mathrm{Ag} N \mathrm{NM}$ and medium physicochemical properties in controlling $\mathrm{Ag}$ NM aggregation kinetics ( $\alpha$ and CCC). We found that, the CCC for Ag NMs increases with the decrease in particle size at a fixed surface (zeta) potential. Otherwise, the CCC might increase with the increase in NM size, or might be independent of NM size, for variable surface (zeta potential), depending on the dependence of NM surface charge on their size. The CCC increase with the decrease in buffer (e.g. phosphate and carbonate) concentration. The CCC for Ag NM decrease with light irradiation due to degradation of surface coating and reduction of the magnitude of surface charge. The CCC is independent of NM concentration for pure electrostatic interactions. However, in the presence of chemical constituents of high affinity to NMs (e.g. Ag NMs in the presence of cystine, carbonate and phosphate anions) that chemisorb on the surface of NMs, the CCC is NM concentration-dependent.

Fully coated, sterically stabilized Ag NMs do not aggregate even in high ionic strength solutions. However, the stability (or CCC) of partially coated Ag NMs decreases with the decrease in surface coverage by the capping agent. Also, sterically stabilized Ag NMs can be destabilized by surface coating replacement, rendering such particles subject to aggregation.

Clearly, the stability of NMs is a complex issue that cannot be described by an individual parameter such as ionic strength, or concentration of NOM, that has been the focus of a significant number of studies in the literature. NM stability is rather determined by a combination of NM and medium physicochemical properties. Chemical interactions of NMs with water constituents such as buffer, 
anions, cystine, etc. play an equally important role in controlling NM stability, especially at environmentally relevant NM concentrations ( $c a$. $\mathrm{ng}$ to $\mu \mathrm{g} \mathrm{L}^{-1}$ range). Therefore, focusing on the physical interactions (e.g. attraction and repulsion) of NM only when taking into account water chemistry in modeling environmental fate and behavior of $\mathrm{NMs}^{88}$ might lead to erroneous conclusions. Overall, these findings are important to underpin nanomaterial risk assessment and environmental fate and behavior studies.

\section{Acknowledgement}

We acknowledge funding support from the National Science Foundation (NSF, 1437307, 1553909), the SmartState Centre for Environmental Nanoscience and Risk, and the Arnold School of Public Health at the University of South Carolina.

\section{Supporting information}

A table of CCC values of Ag NMs in the literature together with the properties of Ag NMs and the media. A Tables of Spearman's correlation tests between CCC (mM) and Z-average diameter ( $\mathrm{nm}), \mathrm{N}$-average diameter $(\mathrm{nm})$ and buffer concentration $(\mathrm{mM})$, respectively, under various experimental conditions 
(1) Klaine, S. J.; Alvarez, P. J. J.; Batley, G. E.; Fernandes, T. F.; Handy, R. D.; Lyon, D. Y.; Mahendra, S.; McLaughlin, M. J.; Lead, J. L. Nanomaterials in the Environment : Behaviour, Fate, Bioavailability and Effects. Environ. Toxicol. Chem. 2008, 27 (9), 1825-1851.

(2) Peijnenburg, W. J.; Baalousha, M.; Chen, J.; Chaudry, Q.; von der Kammer, F.; Kuhlbusch, T. A.; Lead, J.; Nickel, C.; Quik, J. T.; Renker, M. A Review of the Properties and Processes Determining the Fate of Engineered Nanomaterials in the Aquatic Environment. Crit. Rev. Environ. Sci. Technol. 2015, 45 (19), 2084-2134.

(3) Dale, A. L.; Lowry, G. V.; Casman, E. A. Much Ado About $\alpha$ : Reframing the Debate Over Appropriate Fate Descriptors in Nanoparticle Environmental Risk Modeling. Environmental Science: Nano 2015, 2 ( 27-32.

(4) Dale, A. L.; Lowry, G. V.; Casman, E. A. Modeling Nanosilver Transformations in Freshwater Sediments. Environ. Sci. Technol. 2013, 47 (22), 12920-12928.

(5) Baalousha, M.; Cornelis, G.; Kuhlbusch, T. A. J.; Lynch, I.; Nickel, C.; Peijnenburg, W.; van den Brink, N. W. Modeling Nanomaterial Fate and Uptake in the Environment: Current Knowledge and Future Trends. Environ. Sci. Nano. 2016, 3 (2), 323-345.

(6) Dale, A. L.; Casman, E. A.; Lowry, G. V.; Lead, J. R.; Viparelli, E.; Baalousha, M. Modeling Nanomaterial Environmental Fate in Aquatic Systems. Environ. Sci. Technol. 2015, 49 (5), 2587-2593.

(7) Tadros, T. General Principles of Colloid Stability and the Role of Surface Forces. Colloid stability part I. Viley-VCH Verlag GmbH, Weinheim 2007.

(8) Derjaguin, B. V.; Landau, L. D. Theory of the Stability of Strongly Charged Lyophobic Sols and of the Adhesion of Strongly Charged Particles in Solutions of Electrolytes. Acta physicochim. U.R. S. S. 1941, 14 ( 633-662.

(9) Verwey, E. J. W.; Overbeek, J. Th. G. Theory of the Stability of Lyophobic Colloids; Elsevier: Amsterdam, 1948.

(10) Baalousha, M.; Lead, J. R.; Ju-Nam, Y. 3.05 - Natural Colloids and Manufactured Nanoparticles in Aquatic and Terrestrial Systems. In Treatise on Water Science; Wilderer, P., Ed.; Elsevier: Oxford, 2011.

(11) Liu, L.; Moreno, L.; Neretnieks, I. A Novel Approach to Determine the Critical Coagulation Concentration of a Colloidal Dispersion With Plate-Like Particles. Langmuir 2009, 25 (2), 688697.

(12) Hotze, E. M.; Phenrat, T.; Lowry, G. V. Nanoparticle Aggregation: Challenges to Understanding Transport and Reactivity in the Environment. J. Environ. Qual. 2010, 39 (6), 1909-1924. 
(13) Zhou, D.; Keller, A. A. Role of Morphology in the Aggregation Kinetics of ZnO Nanoparticles. Wat. Res. 2010, 44 (9), 2948-2956.

(14) Baalousha, M.; Manciulea, A.; Cumberland, S.; Kendall, K.; Lead, J. R. Aggregation and Surface Properties of Iron Oxide Nanoparticles; Influence of PH and Natural Organic Matter. Environ. Toxicol. Chem. 2008, 27 (9), 1875-1882.

(15) Baalousha, M. Aggregation and Disaggregation of Iron Oxide Nanoparticles: Influence of Particle Concentration, PH and Natural Organic Matter. Sci. Tot. Environ. 2009, 407 (6), 20932101.

(16) Baalousha, M.; Nur, Y.; Römer, I.; Tejamaya, M.; Lead, J. R. Effect of Monovalent and Divalent Cations, Anions and Fulvic Acid on Aggregation of Citrate-Coated Silver Nanoparticles. Sci. Tot. Environ. 2013, 454-455 (0), 119-131.

(17) Penners, N. H. G.; Koopal, L. K. The Effect of Particle Size on the Stability of Haematite ( $\alpha$ Fe2O3) Hydrosols. Colloid Surf. 1987, 28 (67-83.

(18) Zhou, D.; Ji, Z.; Jiang, X.; Dunphy, D. R.; Brinker, J.; Keller, A. A. Influence of Material Properties on TiO 2 Nanoparticle Agglomeration. PLoS ONE 2013, 8 (11), e81239.

(19) Stemig, A. M.; Do, T. A.; Yuwono, V. M.; Arnold, W. A.; Penn, R. L. Goethite Nanoparticle Aggregation: Effects of Buffers, Metal Ions, and 4-Chloronitrobenzene Reduction. Environ. Sci. Nano. 2014, 1 (5), 478-487.

(20) He, Y. T.; Wan, J.; Tokunaga, T. Kinetic Stability of Hematite Nanoparticles: the Effect of Particle Sizes. J. Nanopart. Res. 2008, 10 ( 321-332.

(21) Mulvihill, M. J.; Habas, S. E.; Jen-La Plante, I.; Wan, J.; Mokari, T. Influence of Size, Shape, and Surface Coating on the Stability of Aqueous Suspensions of CdSe Nanoparticles. Chem. Mater. 2010, 22 (18), 5251-5257.

(22) Liu, J.; Legros, S.; Ma, G.; Veinot, J. G. C.; von der Kammer, F.; Hofmann, T. Influence of Surface Functionalization and Particle Size on the Aggregation Kinetics of Engineered Nanoparticles. Chemosphere 2012, 87 (8), 918-924.

(23) Liu, X.; Chen, G.; Su, C. Effects of Material Properties on Sedimentation and Aggregation of Titanium Dioxide Nanoparticles of Anatase and Rutile in the Aqueous Phase. J. Colloid Interf. Sci. 2011, 363 (1), 84-91.

(24) Römer, I.; White, T. A.; Baalousha, M.; Chipman, K.; Viant, M. R.; Lead, J. R. Aggregation and Dispersion of Silver Nanoparticles in Exposure Media for Aquatic Toxicity Tests. J. Chromatogr. A. 2011, 1218 (27), 4226-4233.

(25) Afshinnia, K.; Gibson, I.; Merrifield, R.; Baalousha, M. The Concentration-Dependent Aggregation of Ag NPs Induced by Cystine. Sci. Tot. Environ. 2016, 557-558 (395-403.

(26) Elimelech, M.; Gregory, J.; Jia, X.; Williams, R. A. Particle Deposition and Aggregation: Measurement, Modeling and Simulation.; Butterworth-Heinemann: Oxford, 1995. 
(27) Christian, P.; Von der Kammer, F.; Baalousha, M.; Hofmann, Th. Nanoparticles: Structure, Properties, Preparation and Behaviour in Environmental Media. Environ. Toxicol. Chem. 2008, 17 ( 326-343.

(28) Zhang, H.; Smith, J. A.; Oyanedel-Craver, V. The Effect of Natural Water Conditions on the AntiBacterial Performance and Stability of Silver Nanoparticles Capped With Different Polymers. Wat. Res. 2012, 46 (3), 691-699.

(29) El Badawy, A. M.; Scheckel, K. G.; Suidan, M.; Tolaymat, T. The Impact of Stabilization Mechanism on the Aggregation Kinetics of Silver Nanoparticles. Sci. Tot. Environ. 2012, 429 (1), 325-331.

(30) Huynh, K. A.; Chen, K. L. Aggregation Kinetics of Citrate and Polyvinylpyrrolidone Coated Silver Nanoparticles in Monovalent and Divalent Electrolyte Solutions. Environ. Sci. Technol. 2011, 45 (13), 5564-5571.

(31) Afshinnia, K.; Gibson, I.; Merrifield, R.; Baalousha, M. The Concentration-Dependent Aggregation of Ag NPs Induced by Cystine. Sci. Tot. Environ. 2016.

(32) Lodeiro, P.; Achterberg, E. P.; Pampin, J.; Affatati, A.; El-Shahawi, M. S. Silver Nanoparticles Coated With Natural Polysaccharides As Models to Study AgNP Aggregation Kinetics Using UVVisible Spectrophotometry Upon Discharge in Complex Environments. Sci. Tot. Environ. 2016, 539 ( 7-16.

(33) Lin, S.; Cheng, Y.; Liu, J.; Wiesner, M. R. Polymeric Coatings on Silver Nanoparticles Hinder Autoaggregation but Enhance Attachment to Uncoated Surfaces. Langmuir 2012, 28 (9), 41784186.

(34) Matijevic, E.; Allen, L. H. Interactions of Colloidal Dispersions With Electrolytes. Environ. Sci. Technol. 1969, 3 (3), 264-268.

(35) Hall, S. B.; Duffield, J. R.; Williams, D. R. A Reassessment of the Applicability of the DLVO Theory As an Explanation for the Schulze-Hardy Rule for Colloid Aggregation. J. Colloid Interf. Sci. 1991, 143 (2), 411-415.

(36) Nowicki, W.; Nowicka, G. Verification of the Schulze-Hardy Rule: A Colloid Chemistry Experiment. J. Chem. Ed. 1994, 71 (7), 624.

(37) Verrall, K. E.; Warwick, P.; Fairhurst, A. J. Application of the Schulze-Hardy Rule to Haematite and Haematite/Humate Colloid Stability. Colloids Surf. A: Physicochem. Eng. Aspects. 1999, $150(1-3), 261-273$.

(38) Li, X.; Lenhart, J. J.; Walker, H. W. Aggregation Kinetics and Dissolution of Coated Silver Nanoparticles. Langmuir 2012, 28 (2), 1095-1104.

(39) Li, X.; Lenhart, J. J. Aggregation and Dissolution of Silver Nanoparticles in Natural Surface Water. Environ. Sci. Technol. 2012, 46 (10), 5378-5386. 
(40) Li, X.; Lenhart, J. J.; Walker, H. W. Dissolution-Accompanied Aggregation Kinetics of Silver Nanoparticles. Langmuir 2010, 26 (22), 16690-16698.

(41) Lin, D.; Ma, S.; Zhou, K.; Wu, F.; Yang, K. The Effect of Water Chemistry on Homoaggregations of Various Nanoparticles: Specific Role of $\mathrm{Cl}^{-}$Ions. J. Colloid Interf. Sci. 2015, 450 ( 272-278.

(42) Diegoli, S.; Manciulea, A. L.; Begum, S.; Jones, I. P.; Lead, J. R.; Preece, J. A. Interaction Between Manufactured Gold Nanoparticles and Naturally Occurring Organic Macromolecules. Sci. Tot. Environ. 2008, 402 (1), 51-61.

(43) Amal, R.; Raper, J. A.; Waite, T. D. Effect of Fulvic Acid Adsorption on the Aggregation Kinetics and Structure of Hematite Particles. J. Colloid Interf. Sci. 1992, 151 (1), 244-257.

(44) Frens, G. Particle Size and Sol Stability in Metal Colloids. Kolloid-Zeitschrift und Zeitschrift für Polymere 1972, 250 (7), 736-741.

(45) Shulepov, S. Y.; Frens, G. Surface Roughness and the Particle Size Effect on the Rate of Slow, Perikinetic Coagulation. J. Colloid Interf. Sci. 1995, 170 (1), 44-49.

(46) Watillon, A.; Joseph-Petit, A. M. Interactions Between Spherical Particles of Monodisperse Polystyrene Latices. Discuss. Faraday Soc. 1966, 42 ( 143-153.

(47) Matthews, B. A.; Rhodes, C. T. The Use of the Coulter Counter for Investigating the Coagulation Kinetics of Mixed Monodisperse Particulate Systems. J. Colloid Interf. Sci. 1968, 28 (1), 71-81.

(48) Iler, R. K. Coagulation of Colloidal Silica by Calcium Ions, Mechanism, and Effect of Particle Size. J. Colloid Interf. Sci. 1975, 53 (3), 476-488.

(49) Kotera, A.; Furusawa, K.; Kudo, M. K. Colloid Chemical Studies of Polystyrene Latices Polymerized Without Any Surface-Active Agents. Kolloid-Zeitschrift und Zeitschrift für Polymere 1970, 240 (1-2), 837-842.

(50) Ottewill, R. H.; Shaw, J. N. Stability of Monodisperse Polystyrene Latex Dispersions of Various Sizes. Discussions of the Faraday Society 1966, 42 (0), 154-163.

(51) Elimelech, M.; O'Melia, C. R. Effect of Particle Size on Collision Efficiency in the Deposition of Brownian Particles With Electrostatic Energy Barriers. Langmuir 1990, 6 (6), 1153-1163.

(52) Gedan, H.; Lichtenfeld, H.; Sonntag, H. Some Comments on Von Smoluchowski Theory of Rapid Coagulation. Colloid Polymer Sci. 1982, 260 (12), 1151-1152.

(53) Wiese, G. R.; Healy, T. W. Effect of Particle Size on Colloid Stability. Trans. Faraday Soc. 1970, 66 ( $490-499$.

(54) Molina-Bolívar, J. A.; Galisteo-González, F.; Hidalgo-Alvarez, R. Repeptization Determined by Turbidity and Photon Correlation Spectroscopy Measurements: Particle Size Effects. J. Colloid Interf. Sci. 1997, 195 (2), 289-298. 
(55) Schenkel, J. H.; Kitchener, J. A. A Test of the Derjaguin-Verwey-Overbeek Theory With a Colloidal Suspension. Trans. Faraday Soc. 1960, 56 ( 161-173.

(56) Marshall, J. K.; Kitchener, J. A. The Deposition of Colloidal Particles on Smooth Solids. J. Colloid Interf. Sci. 1966, 22 (4), 342-351.

(57) Hsu, J. P.; Liu, B. T. Effect of Particle Size on Critical Coagulation Concentration. J. Colloid Interf. Sci. 1998, 198 (1), 186-189.

(58) Buchholz, A.; Laskov, C.; Haderlein, S. B. Effects of Zwitterionic Buffers on Sorption of Ferrous Iron at Goethite and Its Oxidation by CCl4. Environ. Sci. Technol. 2011, 45 (8), 3355-3360.

(59) Liang, L. Effects of Surface Hemistry on Kinetics of Coagulation of Submicron Iron-Oxide Particles (Alpha-Iron-Oxide) in Water. 1988.

(60) Piccapietra, F.; Sigg, L.; Behra, R. Colloidal Stability of Carbonate Coated Silver Nanoparticles in Synthetic and Natural Freshwater. Environ. Sci. Technol. 2012, 46 (2), 818-825.

(61) Metreveli, G.; Frombold, B.; Seitz, F.; Grun, A.; Philippe, A.; Rosenfeldt, R. R.; Bundschuh, M.; Schulz, R.; Manz, W.; Schaumann, G. E. Impact of Chemical Composition of Ecotoxicological Test Media on the Stability and Aggregation Status of Silver Nanoparticles. Environ. Sci. Nano. 2016.

(62) Pokhrel, L. R.; Dubey, B.; Scheuerman, P. R. Impacts of Select Organic Ligands on the Colloidal Stability, Dissolution Dynamics, and Toxicity of Silver Nanoparticles. Environ. Sci. Technol. 2013, 47 (22), 12877-12885.

(63) Wang, F.; Liu, X.; Lu, C. H.; Willner, I. Cysteine-Mediated Aggregation of Au Nanoparticles: The Development of a H2O2 Sensor and Oxidase-Based Biosensors. ACS Nano 2013, 7 (8), 72787286.

(64) Zakaria, H. M.; Shah, A.; Konieczny, M.; Hoffmann, J. A.; Nijdam, A. J.; Reeves, M. E. Small Molecule- and Amino Acid-Induced Aggregation of Gold Nanoparticles. Langmuir 2013, 29 (25), 7661-7673.

(65) Doyen, M.; Goole, J.; Bartik, K.; Bruylants, G. Amino Acid Induced Fractal Aggregation of Gold Nanoparticles: Why and How. J. Colloid Interf. Sci. 2016, 464 ( 160-166.

(66) Chegel, V.; Rachkov, O.; Lopatynskyi, A.; Ishihara, S.; Yanchuk, I.; Nemoto, Y.; Hill, J. P.; Ariga, K. Gold Nanoparticles Aggregation: Drastic Effect of Cooperative Functionalities in a Single Molecular Conjugate. J. Phys. Chem. C. 2012, 116 (4), 2683-2690.

(67) Gebauer, J. S.; Malissek, M.; Simon, S.; Knauer, S. K.; Maskos, M.; Stauber, R. H.; Peukert, W.; Treuel, L. Impact of the Nanoparticle-Protein Corona on Colloidal Stability and Protein Structure. Langmuir 2012, 28 (25), 9673-9679.

(68) Lesniak, A.; Salvati, A.; Santos-Martinez, M. J.; Radomski, M. W.; Dawson, K. A.; +àberg, C. Nanoparticle Adhesion to the Cell Membrane and Its Effect on Nanoparticle Uptake Efficiency. J. Am. Chem. Soc. 2013, 135 (4), 1438-1444. 
(69) Matijevic, E.; Stryker, L. J. Coagulation and Reversal of Charge of Lyophobic Colloids by Hydrolyzed Metal Ions. J. Colloid Interf. Sci. 1966, 22 (1), 68-77.

(70) Stryker, L. J.; Matijevic, E. Counterion Complexing and Sol Stability. II. Coagulation Effects of Aluminum Sulfate in Acidic Solutions. J. Phys. Chem. 1969, 73 (5), 1484-1487.

(71) Zhang, W.; Yao, Y.; Li, K.; Huang, Y.; Chen, Y. Influence of Dissolved Oxygen on Aggregation Kinetics of Citrate-Coated Silver Nanoparticles. Environ. Pollut. 2011, 159 (12), 3757-3762.

(72) Li, Y.; Zhang, W.; Li, K.; Yao, Y.; Niu, J.; Chen, Y. Oxidative Dissolution of Polymer-Coated $\mathrm{CdSe} / \mathrm{ZnS}$ Quantum Dots Under UV Irradiation: Mechanisms and Kinetics. Environ. Pollut. 2012, 164 ( 259-266.

(73) Zhang, W.; Yao, Y.; Sullivan, N.; Chen, Y. Modeling the Primary Size Effects of Citrate-Coated Silver Nanoparticles on Their Ion Release Kinetics. Environ. Sci. Technol. 2011, 45 (10), 44224428.

(74) Liu, J.; Hurt, R. H. Ion Release Kinetics and Particle Persistence in Aqueous Nano-Silver Colloids. Environ. Sci. Technol. 2010, 44 (6), 2169-2175.

(75) Römer, I.; Wang, Z. W.; Merrifield, R. C.; Palmer, R. E.; Lead, J. High Resolution STEM-EELS Study of Silver Nanoparticles Exposed to Light and Humic Substances. Environ. Sci. Technol. 2016, 50 (5), 2183-2190.

(76) Ho, C. M.; Yau, S. K.-W.; Lok, C. N.; So, M. H.; Che, C. M. Oxidative Dissolution of Silver Nanoparticles by Biologically Relevant Oxidants: A Kinetic and Mechanistic Study. Chem. Asian J. 2010, 5 (2), 285-293.

(77) Li, Y.; Zhang, W.; Niu, J.; Chen, Y. Surface-Coating-Dependent Dissolution, Aggregation, and Reactive Oxygen Species (ROS) Generation of Silver Nanoparticles Under Different Irradiation Conditions. Environ. Sci. Technol. 2013.

(78) Misra, N.; Kumar, V.; Borde, L.; Varshney, L. Localized Surface Plasmon Resonance-Optical Sensors Based on Radiolytically Synthesized Silver Nanoparticles for Estimation of Uric Acid. Sens. Actuat. B: Chem. 2013, 178 ( 371-378.

(79) Correa-Duarte, M. A.; Giersig, M.; Liz-Marzán, L. M. Stabilization of CdS Semiconductor Nanoparticles Against Photodegradation by a Silica Coating Procedure. Chem. Phys. Lett. 1998, 286 (5ГÇô6), 497-501.

(80) Gorham, J. M.; MacCuspie, R. I.; Klein, K. L.; Fairbrother, D. H.; Holbrook, D. UV-Induced Photochemical Transformations of Citrate-Capped Silver Nanoparticle Suspensions. J. Nanopart. Res. 2012, 14 (10).

(81) Shi, J. P.; Ma, C. Y.; Xu, B.; Zhang, H. W.; Yu, C. P. Effect of Light on Toxicity of Nanosilver to Tetrahymena Pyriformis. Environ. Toxicol. Chem. 2012, 31 (7), 1630-1638.

(82) Cheng, Y.; Yin, L.; Lin, S.; Wiesner, M.; Bernhardt, E.; Liu, J. Toxicity Reduction of PolymerStabilized Silver Nanoparticles by Sunlight. J. Phys. Chem. C. 2011, 115 (11), 4425-4432. 
(83) Mittelman, A. M.; Fortner, J. D.; Pennell, K. D. Effects of Ultraviolet Light on Silver Nanoparticle Mobility and Dissolution. Environ. Sci. Nano. 2015, 2 (6), 683-691.

(84) Shi, J.; Xu, B.; Sun, X.; Ma, C.; Yu, C.; Zhang, H. Light Induced Toxicity Reduction of Silver Nanoparticles to Tetrahymena Pyriformis: Effect of Particle Size. Aquat. Toxicol. 2013, 132-133 (53-60.

(85) Tiraferri, A.; Chen, K. L.; Sethi, R.; Elimelech, M. Reduced Aggregation and Sedimentation of Zero-Valent Iron Nanoparticles in the Presence of Guar Gum. J. Colloid Interf. Sci. 2008, 324 (1-2), 71-79.

(86) Tejamaya, M.; Römer, I.; Merrifield, R. C.; Lead, J. R. Stability of Citrate, PVP, and PEG Coated Silver Nanoparticles in Ecotoxicology Media. Environ. Sci. Technol. 2012, 46 (13), 7011-7017.

(87) Jayme, M. L.; Dunstan, D. E.; Gee, M. L. Zeta Potentials of Gum Arabic Stabilised Oil in Water Emulsions. Food Hydrocolloids 1999, 13 (6), 459-465.

(88) Sani-Kast, N.; Scheringer, M.; Slomberg, D.; Labille, J.; Praetorius, A.; Ollivier, P.; Hungerbühler, $\mathrm{K}$. Addressing the Complexity of Water Chemistry in Environmental Fate Modeling for Engineered Nanoparticles. Sci. Tot. Environ. 2015. 

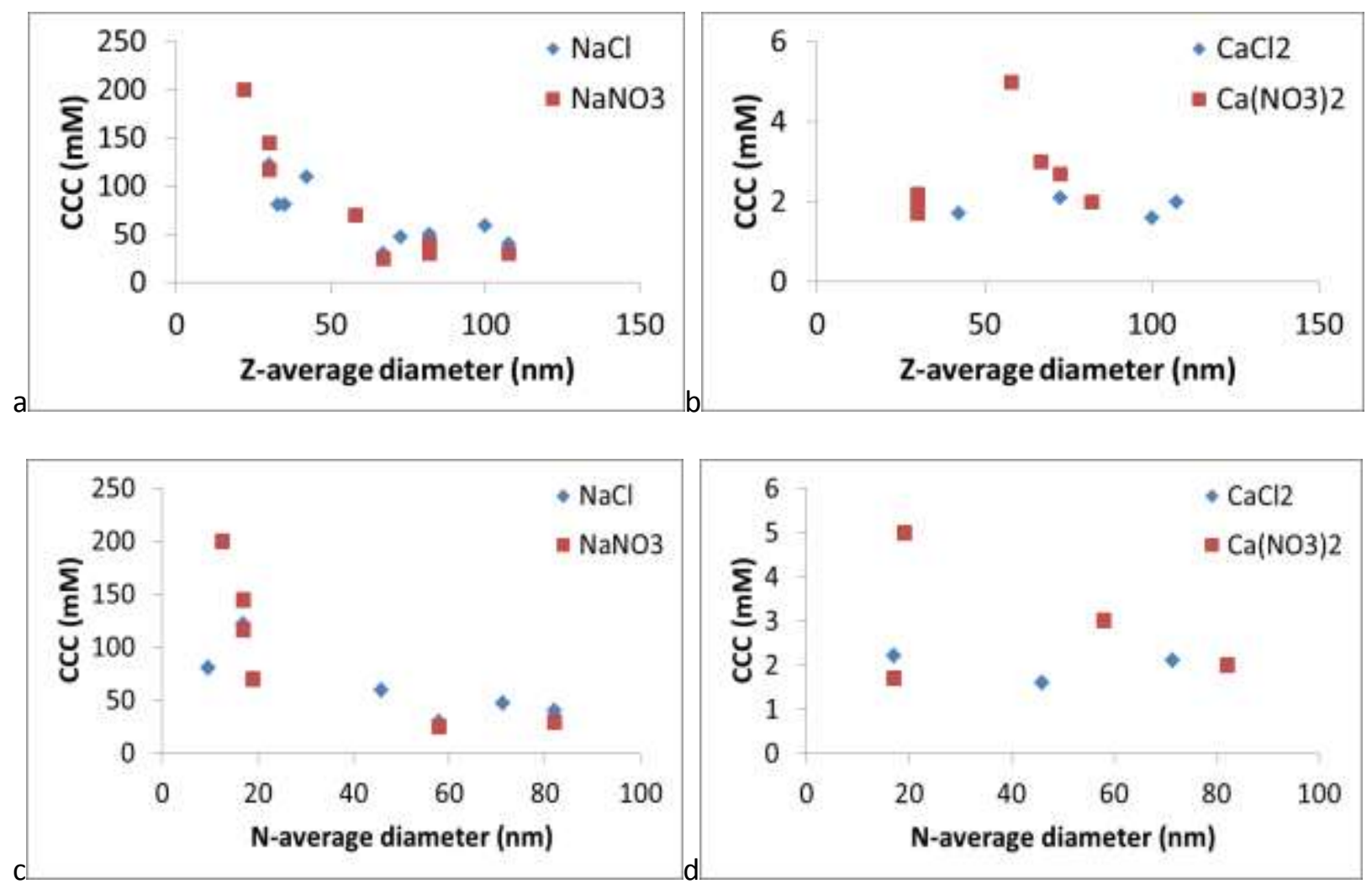

Figure 1. Dependence of CCC for charge stabilized Ag NMs on (a, b) z-average hydrodynamic diameter, and (c, d) N-average diameter for ( $a, c)$ monovalent counter ions, and (b, d) divalent counter ions. 


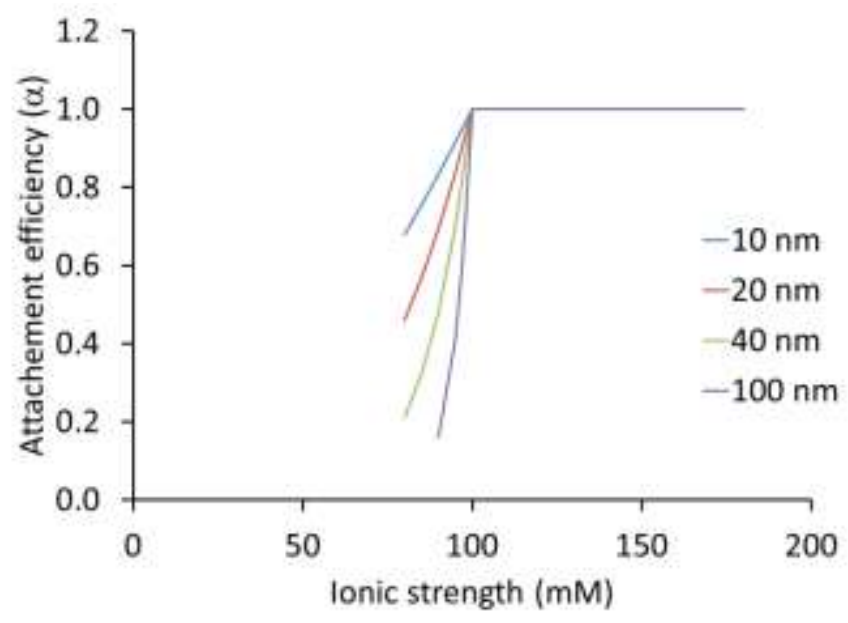

Figure 2. Dependence of the attachment efficiency and CCC on the NM size according to DLVO theory. A $=3.7 \times 10^{-20} \mathrm{~J}$, zeta potential assumed constant $-42 \mathrm{mV}$ for all ionic strength conditions for simplification. This figure is used for illustrative purposes and is not meant to be an accurate calculation of the $\alpha$-ionic strength profile. 


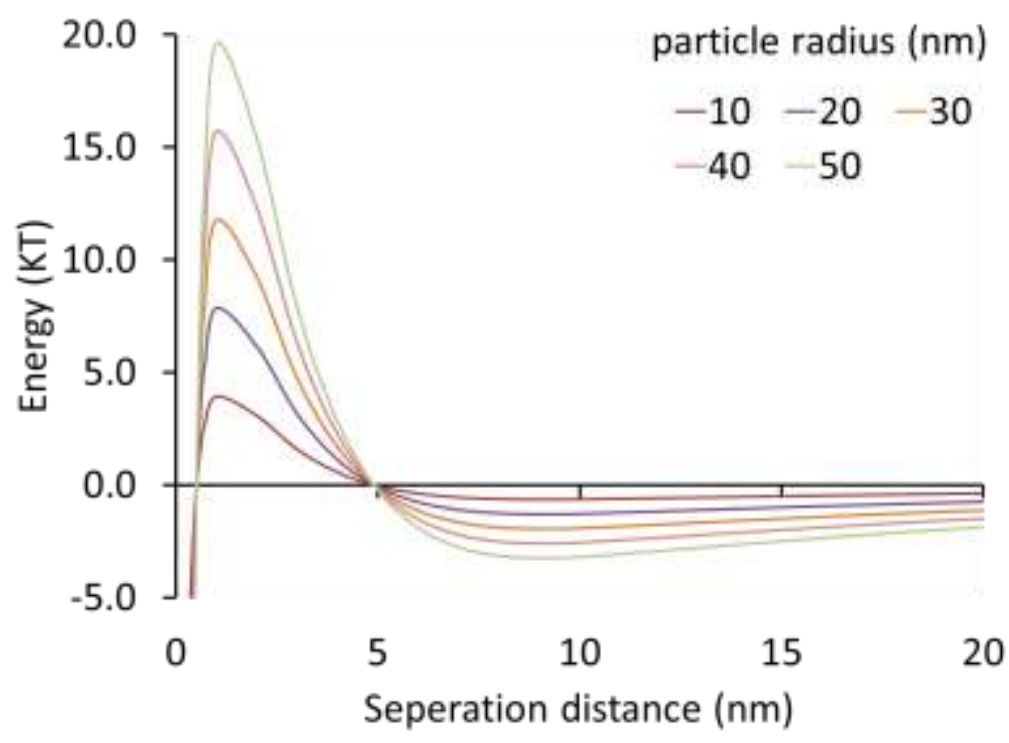

Figure 3. Dependence of secondary minimum depth on NM size according to classical DLVO theory calculated using a Hamaker constant of $3.7 \times 10^{-20} \mathrm{~J}$, assuming a constant zeta potential of $-42 \mathrm{mV}$, and IS of $30 \mathrm{mM}$. 


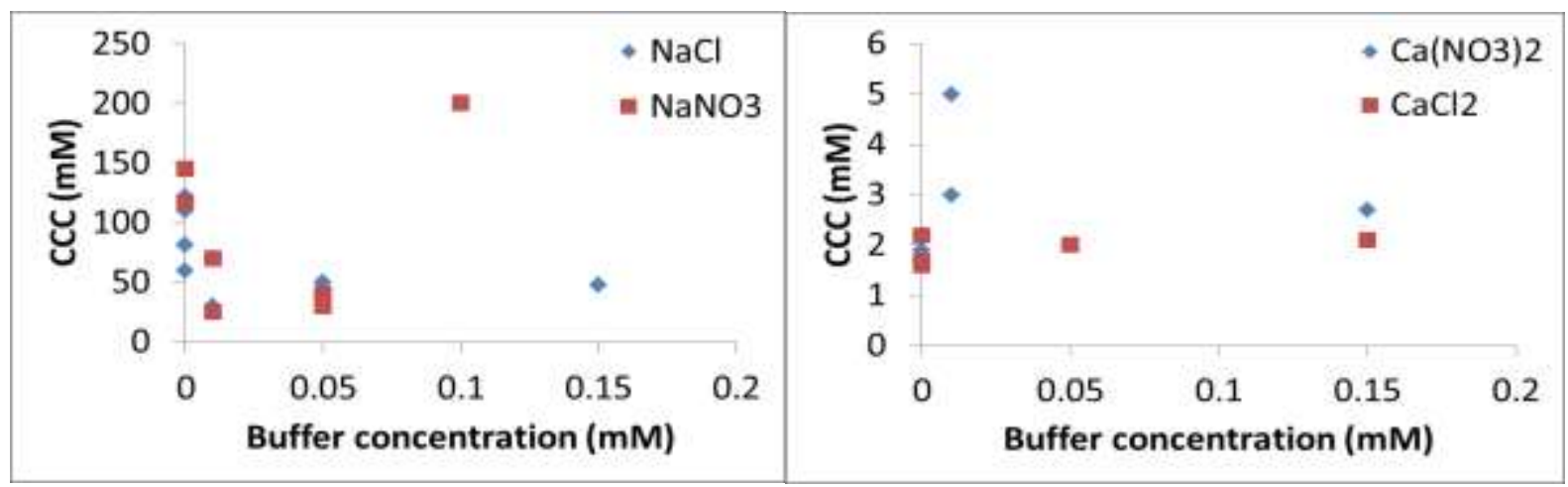

Figure 4. Dependence of CCC for charge stabilized Ag NMs on buffer concentration for (a) monovalent counterions, and (b) divalent counter ions 


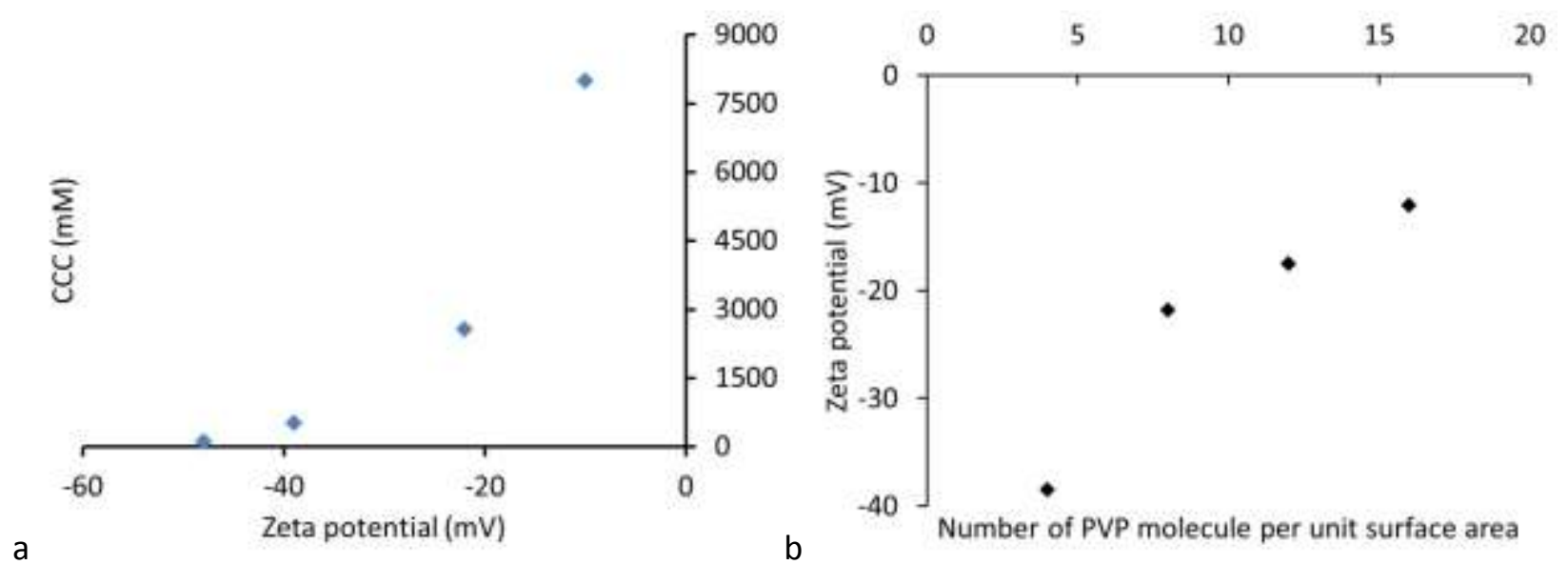

Figure 5. (a) CCC of PVP coated-Ag NMs as a function of zeta potential of as synthesized PVP-Ag NMs (data collected from the literature). For the highest CCC value, CCC was assumed to be the highest concentration of electrolyte investigated for illustration purposes, but no CCC was measured ${ }^{29}$, and (b) zeta potential of citrate-Ag NMs as a function of number of PVP molecules per $\mathrm{nm}^{2}$ surface area, following citrate replacement by PVP using ligand exchange approach (experiments performed by authors). 

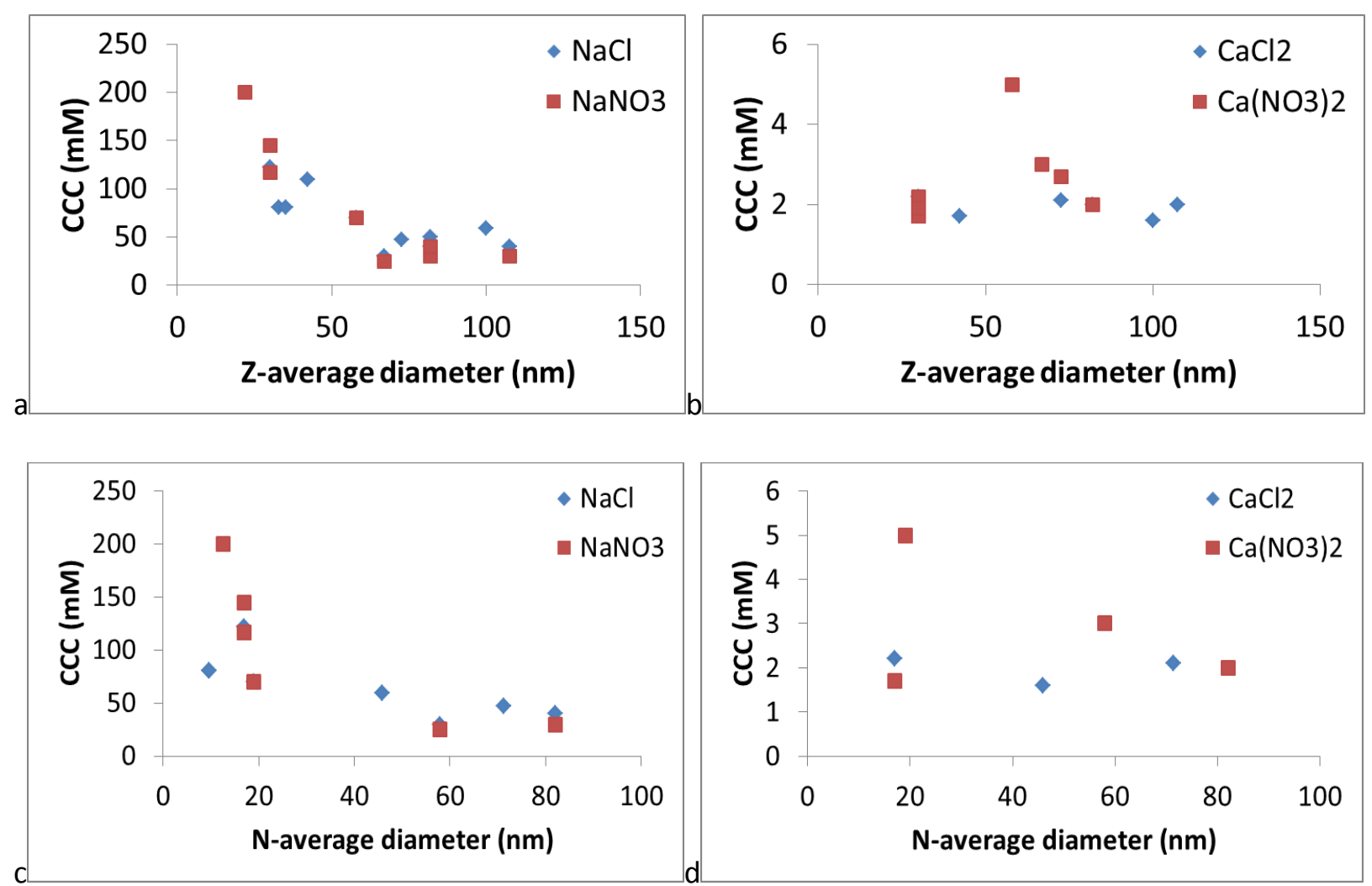

Figure 1. Dependence of CCC for charge stabilized Ag NMs on $(a, b)$-average hydrodynamic diameter, and $(c, d) \mathrm{N}$-average diameter for $(\mathrm{a}, \mathrm{c})$ monovalent counter ions, and $(\mathrm{b}, \mathrm{d})$ divalent counter ions. 
Figure 2. Dependence of the attachment efficiency and CCC on the NM size according to DLVO theory. A $=3.7 \times 10^{-20} \mathrm{~J}$, zeta potential assumed constant $-42 \mathrm{mV}$ for all ionic strength conditions for simplification. This figure is used for illustrative purposes and is not meant to be an accurate calculation of the $\alpha$-ionic strength profile. 


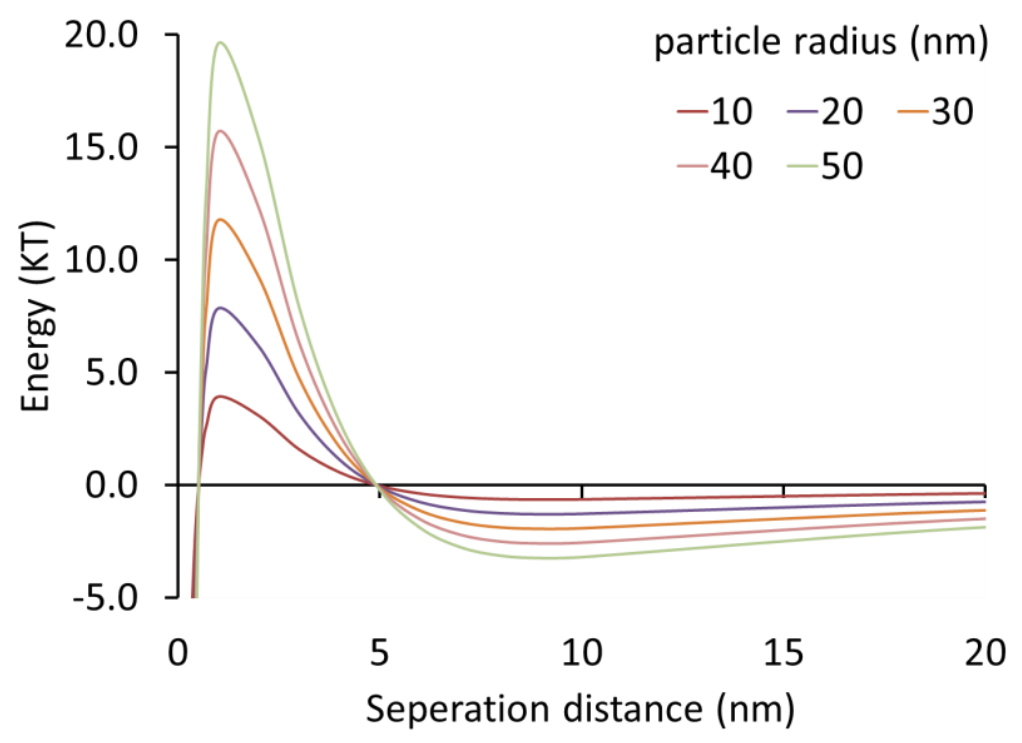

Figure 3. Dependence of secondary minimum depth on NM size according to classical DLVO theory calculated using a Hamaker constant of $3.7 \times 10^{-20} \mathrm{~J}$, assuming a constant zeta potential of $-42 \mathrm{mV}$, and IS of $30 \mathrm{mM}$. 


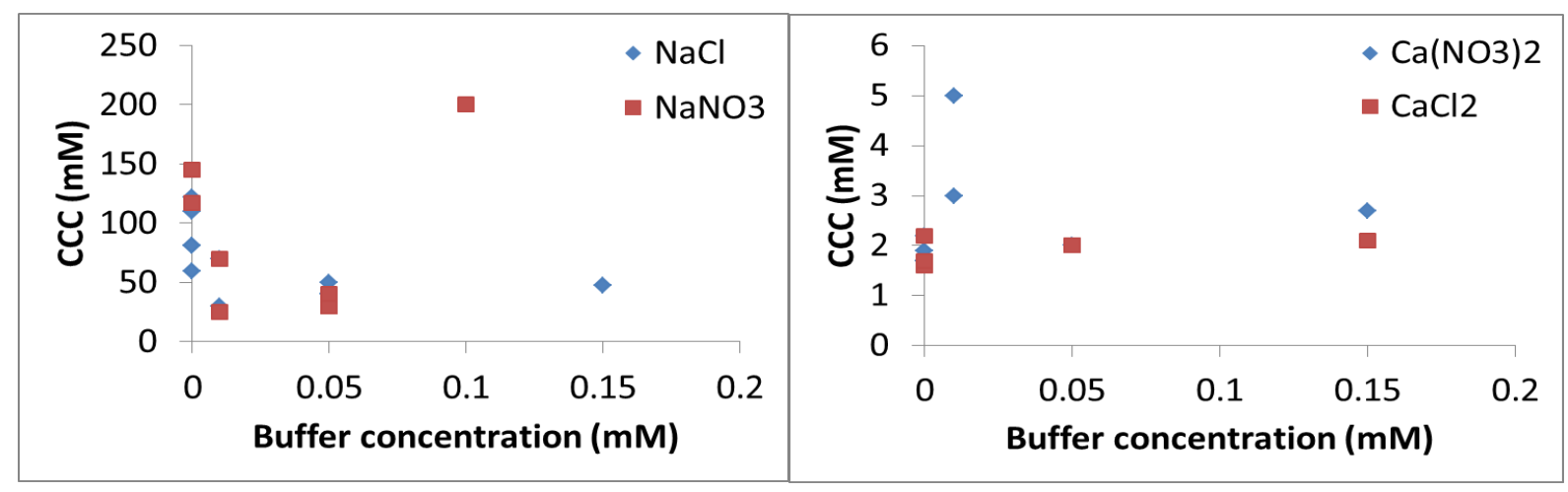

Figure 4. Dependence of CCC for charge stabilized Ag NMs on buffer concentration for (a) monovalent counterions, and (b) divalent counter ions 
a

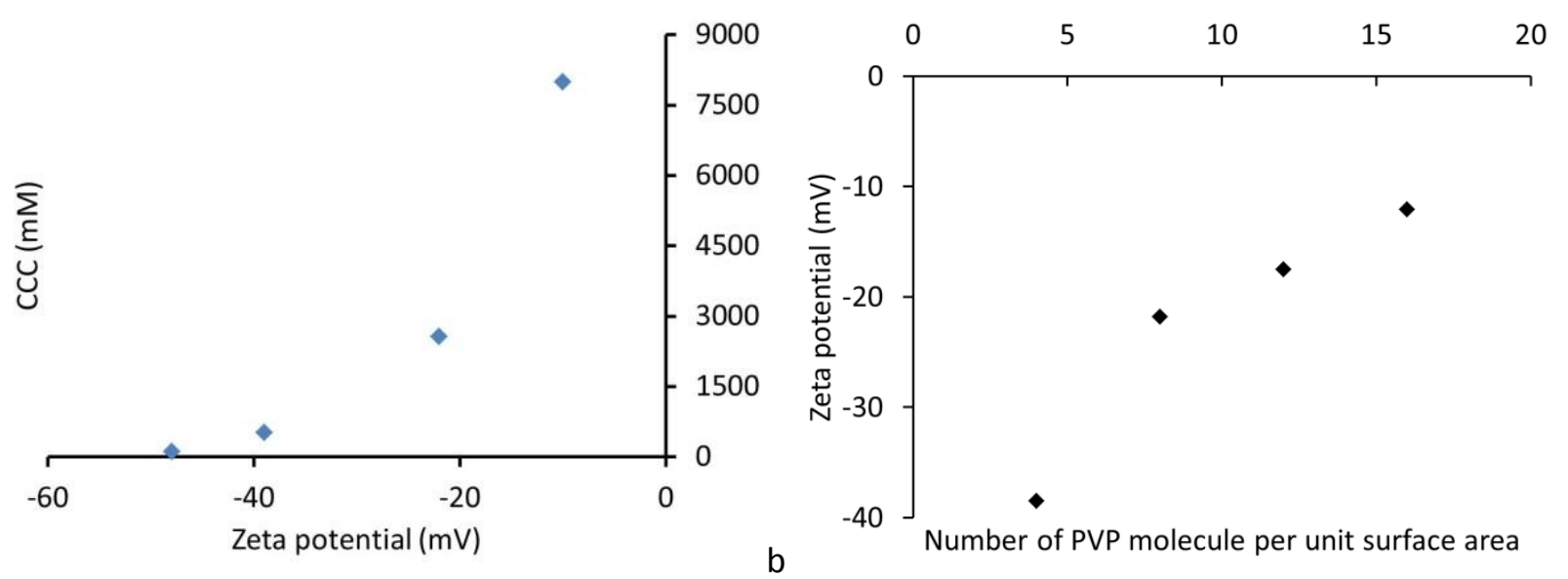

Figure 5. (a) CCC of PVP coated-Ag NMs as a function of zeta potential of as synthesized PVP-Ag NMs (data collected from the literature). For the highest CCC value, CCC was assumed to be the highest concentration of electrolyte investigated for illustration purposes, but no CCC was measured ${ }^{29}$, and (b) zeta potential of citrate-Ag NMs as a function of number of PVP molecules per $\mathrm{nm}^{2}$ surface area, following citrate replacement by PVP using ligand exchange approach (experiments performed by authors). 